Fermilab-Pub-97/227-T

TAUP 2409-97

Revised 7 April 1998

\title{
Representation of Quantum Mechanical Resonances in the Lax-Phillips Hilbert Space
}

\author{
Y. Strauss ${ }^{\dagger}$ and L.P. Horwitz ${ }^{* \dagger, \ddagger}$ \\ E. Eisenberg ${ }^{\ddagger}$ \\ * Fermi National Accelerator Laboratory \\ Box 500 Batavia, IL 60510 \\ ${ }^{\dagger}$ School of Physics, Tel Aviv University \\ Raymond and Beverly Sackler Faculty of Exact Sciences \\ Ramat Aviv, 69978 Israel \\ ${ }^{\ddagger}$ Department of Physics \\ Bar Ilan University \\ Ramat Gan, 59500 Israel
}

\begin{abstract}
We discuss the quantum Lax-Phillips theory of scattering and unstable systems. In this framework, the decay of an unstable system is described by a semigroup. The spectrum of the generator of the semigroup corresponds to the singularities of the LaxPhillips $S$-matrix. In the case of discrete (complex) spectrum of the generator of the semigroup, associated with resonances, the decay law is exactly exponential. The states corresponding to these resonances (eigenfunctions of the generator of the semigroup) lie in the Lax-Phillips Hilbert space, and therefore all physical properties of the resonant states can be computed. We show that the Lax-Phillips $S$-matrix is unitarily related to the $S$-matrix of standard scattering theory by a unitary transformation parametrized by the spectral variable $\sigma$ of the Lax-Phillips theory. Analytic continuation in $\sigma$ has some of the properties of a method developed some time ago for application to dilation analytic potentials. We work out an illustrative example using a Lee-Friedrichs model for the underlying dynamical system.
\end{abstract}




\section{Introduction}

There has been considerable effort in recent years in the development of the theoretical framework of Lax and Phillips scattering theory ${ }^{1}$ for the description of quantum mechanical systems $^{2,3,4}$. This work is motivated by the requirement that the decay law of a decaying system should be exactly exponential if the simple idea that a set of independent unstable systems consists of a population for which each element has a probability, say $\Gamma$, to decay, per unit time. The resulting exponential law $\left(\propto e^{-\Gamma t}\right)$ corresponds to an exact semigroup evolution of the state in the underlying Hilbert space, defined as a family of bounded operators on that space satisfying

$$
Z\left(t_{1}\right) Z\left(t_{2}\right)=Z\left(t_{1}+t_{2}\right)
$$

where $t_{1}, t_{2} \geq 0$, and $Z(t)$ may have no inverse. If the decay of an unstable system is to be associated with an irreversible process, then its evolution necessarily has the property (1.1). ${ }^{5}$ The standard model of Wigner and Weisskopf ${ }^{6}$, based on the computation of the survival amplitude $A(t)$ as the scalar product

$$
A(t)=\left(\psi, e^{-i H t} \psi\right)
$$

where $\psi$ is the initial state of the unstable system and $H$ is the Hamiltonian for the full evolution, results in a good approximation to an exponential decay law for values of $t$ sufficiently large (Wigner and Weisskopf ${ }^{6}$ calculated an atomic linewidth in this way) but cannot result in a semigroup ${ }^{7}$. * When applied to a two-channel system, such as the decay of the $K^{0}$ meson, one easily sees that the poles of the resolvent for the WignerWeisskopf evolution of the two channel systems result in non-orthogonal residues that generate interference terms, which destroy the semigroup property, to accumulate in the calculation of predictions for regeneration experiments ${ }^{8}$. The Yang- $\mathrm{Wu}^{9}$ parametrization of the $K^{0}$ decay processes, based on a Gamow ${ }^{10}$ type evolution generated by an effective $2 x 2$ non-Hermitian matrix Hamiltonian, on the other hand, results in an evolution that is an exact semigroup. It should be possible, with sufficiently careful experiments, to observe the difference in the two types of predictions. It appears that the phenomenological parametrization of refs. 9, which results in semigroup evolution, is indeed consistent to a high degree of accuracy with the experimental results on $K$-meson decay ${ }^{11}$.

The Wigner-Weisskopf model results in non-semigroup evolution independently of the dynamics of the system. Reversible transitions of a quantum mechanical system, such as adiabatic precession of a magnetic moment or tunneling through a potential barrier ${ }^{12}$, which are not radiative, could be expected to be well-described by the Wigner-Weisskopf formula.

In order to achieve exact exponential decay, methods of analytic extension of the Wigner-Weisskopf model to a generalized space have been studied ${ }^{13}$. The generalized states, occurring in the large sector of a Gel'fand triple, are constructed by defining a bilinear form, and analytically continuing a parameter (energy eigenvalue) in one of the

* This formula is generally applied to the transitions induced by interacting fields on states in the Hilbert space of a quantum field theory as well. 
vectors to achieve an exact complex eigenvalue. Although it is possible to achieve an exact exponential decay in this way, the resulting (Banach space) vector has no properties other than to describe this decay law; one cannot compute other properties of the system in this "state". Identifying some representation of the resonant state, it would be of interest, in some applications, to compute, for example, its localization properties, its momentum distribution, or its mean spin.

The quantum Lax-Phillips theor $y^{2,3}$, constructed by embedding the quantum theory into the original Lax-Phillips scattering theory ${ }^{1}$ (originally developed for hyperbolic systems, such as acoustic or electromagnetic waves), describes the resonance as a state in a Hilbert space, and therefore it is possible, in principle, to calculate all measurable properties of the system in this state. Moreover, the quantum Lax-Phillips theory provides a framework for understanding the decay of an unstable system as an irreversible process. It appears, in fact, that this framework is categorical for the description of irreversible processes.

It is clearly desirable to construct a theory which admits the exact semigroup property, but has sufficient structure to describe non-semigroup behavior as well, according to the dynamical properties of the system. The quantum Lax-Phillips theory contains the latter possibility as well, but in this work, we shall restrict ourselves to a study of the semigroup property, associated with irreversible processes.

The scattering theory of Lax and Phillips assumes the existence of a Hilbert space $\overline{\mathcal{H}}$ of physical states in which there are two distinguished orthogonal subspaces $\mathcal{D}_{+}$and $\mathcal{D}_{-}$ with the properties

$$
\begin{array}{rlrl}
U(\tau) \mathcal{D}_{+} & \subset \mathcal{D}_{+} & \tau>0 \\
U(\tau) \mathcal{D}_{-} & \subset \mathcal{D}_{-} & \tau<0 \\
\bigcap_{\tau} U(\tau) \mathcal{D}_{ \pm} & =\{0\} & \\
\bigcup_{\tau} U(\tau) \mathcal{D}_{ \pm} & =\overline{\mathcal{H}},
\end{array}
$$

i.e., the subspaces $\mathcal{D}_{ \pm}$are stable under the action of the full unitary dynamical evolution $U(\tau)$, a function of the physical laboratory time, for positive and negatives times $\tau$ respectively; over all $\tau$, the evolution operator generates a dense set in $\overline{\mathcal{H}}$ from either $\mathcal{D}_{+}$or $\mathcal{D}_{-}$. We shall call $\mathcal{D}_{+}$the outgoing subspace and $\mathcal{D}_{-}$the incoming subspace with respect to the group $U(\tau)$.

A theorem of Sinai ${ }^{14}$ then assures that $\overline{\mathcal{H}}$ can be represented as a family of Hilbert spaces obtained by foliating $\overline{\mathcal{H}}$ along the real line, which we shall call $\{t\}$, in the form of a direct integral

$$
\overline{\mathcal{H}}=\int_{\oplus} \mathcal{H}_{t},
$$

where the set of auxiliary Hilbert spaces $\mathcal{H}_{t}$ are all isomorphic. Representing these spaces in terms of square-integrable functions, we define the norm in the direct integral space (we use Lesbesgue measure) as

$$
\|f\|^{2}=\int_{-\infty}^{\infty} d t\left\|f_{t}\right\|_{H}^{2}
$$


where $f \in \bar{H}$ represents $\overline{\mathcal{H}}$ in terms of the $L^{2}$ function space $L^{2}(-\infty, \infty, H)$, and $f_{t} \in H$, the $L^{2}$ function space representing $\mathcal{H}_{t}$ for any $t$. The Sinai theorem furthermore asserts that there are representations for which the action of the full evolution group $U(\tau)$ on $L^{2}(-\infty, \infty, H)$ is translation by $\tau$ units. Given $D_{ \pm}$(the $L^{2}$ spaces representing $\left.\mathcal{D}_{ \pm}\right)$, there is such a representation, called the incoming representation ${ }^{1}$, for which functions in $D_{-}$ have support in $L^{2}(-\infty, 0, H)$, and another called the outgoing representation, for which functions in $D_{+}$have support in $L^{2}(0, \infty, H)$.

Lax and Phillips ${ }^{1}$ show that there are unitary operators $W_{ \pm}$, called wave operators, which map elements in $\overline{\mathcal{H}}$, respectively, to these representations. They define an $S$-matrix,

$$
S=W_{+} W_{-}^{-1}
$$

which connects these representations; it is unitary, commutes with translations, and maps $L^{2}(-\infty, 0)$ into itself. The singularities of this $S$-matrix, in what we shall define as the spectral representation, correspond to the spectrum of the generator of the exact semigroup characterizing the evolution of the unstable system.

With the assumptions stated above on the properties of the subspaces $\mathcal{D}_{+}$and $\mathcal{D}_{-}$, Lax and Phillips ${ }^{1}$ prove that the family of operators

$$
Z(\tau) \equiv P_{+} U(\tau) P_{-} \quad(\tau \geq 0)
$$

where $P_{ \pm}$are projections into the orthogonal complements of $\mathcal{D}_{ \pm}$, respectively, is a contractive, continuous, semigroup. This operator annihilates vectors in $\mathcal{D}_{ \pm}$and carries the space

$$
\mathcal{K}=\overline{\mathcal{H}} \ominus \mathcal{D}_{+} \ominus \mathcal{D}_{-}
$$

into itself, with norm tending to zero for every element in $\mathcal{K}$.

The existence of a semigroup law for transitions in the framework of the usual quantum mechanical Hilbert space has been shown to be unattainable ${ }^{7}$. However, Flesia and Piron $^{2}$ found that the direct integral of quantum mechanical Hilbert spaces can provide a framework for the Lax-Phillips construction, resulting in a structure directly analogous to the foliation (1.4). In this construction, it was found ${ }^{4}$ that for the representation in which the free evolution is represented by translation on the foliation parameter in Eq. (1.5) (and for which it is assumed that $D_{ \pm}$have definite support properties), the full evolution of the system must be an integral kernel in order to achieve the connection between the LaxPhillips $S$-matrix and the semigroup. In this work we show that the evolution operator for the physical model for the system may be pointwise, in a representation which we shall call the model representation, but in another representation, corresponding to a different foliation, the necessary conditions for the construction of a non-trivial Lax-Phillips theory can be naturally realized. The natural association of the time parameter in the model representation with the foliation asserted by the theorem of Sinai ${ }^{14}$, as we shall show, does not necessarily correspond to the proper embedding of the quantum theory into the Lax-Phillips framework.

If we identify elements in the space $\overline{\mathcal{H}}$ with physical states, and identify the subspace $\mathcal{K}$ with the unstable system, we see that the quantum Lax Phillips theory provides a 
framework for the description of an unstable system which decays according to a semigroup law. We remark that, taking a vector $\psi_{0}$ in $\mathcal{K}$, and evolving it under the action of $U(\tau)$, the projection back into the original state is

$$
\begin{aligned}
A(\tau) & =\left(\psi_{0}, U(\tau) \psi_{0}\right) \\
& =\left(\psi_{0}, P_{\mathcal{K}} U(\tau) P_{\mathcal{K}} \psi_{0}\right) \\
& =\left(\psi_{0}, Z(\tau) \psi_{0}\right),
\end{aligned}
$$

so that the survival amplitude of the Lax-Phillips theory, analogous to that of the WignerWeisskopf formula (1.2), has the exact exponential behavior. The difference between this result and the corresponding expression (1.2) for the Wigner-Weisskopf theory can be accounted for by the fact that there are translation representations for $U(\tau)$, and that the definition of the subspace $\mathcal{K}$ is related to the support properties along the foliation axis on which these translations are induced. ${ }^{3}$

Functions in the space $\bar{H}$, representing the elements of $\overline{\mathcal{H}}$, depend on the variable $t$ as well as the variables of the auxiliary space $H$. The measure space of this Hilbert space of states is one dimension larger than that of a quantum theory represented in the auxiliary space alone. Identifying this additional variable with an observable (in the sense of a quantum mechanical observable) time, we may understand this representation of a state as a virtual history. The collection of such histories forms a quantum ensemble; the absolute square of the wave function corresponds to the probability that the system would be found, as a result of measurement, at time $t$ in a particular configuration in the auxiliary space (in the state described by this wave function), i.e., an element of one of the virtual histories. For example, the expectation value of the position variable $x$ at a given $t$ is, in the standard interpretation of the auxiliary space as a space of quantum states,

$$
\langle x\rangle_{t}=\frac{\left(\psi_{t}, x \psi_{t}\right)}{\left\|\psi_{t}\right\|^{2}} .
$$

The full expectation value in the physical Lax-Phillips state, according to (1.5), is then ${ }^{4}$

$$
\int d t\left(\psi_{t}, x \psi_{t}\right)=\int d t\left\|\psi_{t}\right\|^{2}\langle x\rangle_{t},
$$

so we see that $\left\|\psi_{t}\right\|^{2}$ corresponds to the probability to find a signal which indicates the presence of the system at the time $t$ (in the same way that $x$ is interpreted as a dynamical variable in the quantum theory).

One may ask, in this framework, which results in a precise semigroup behavior for an unstable system, whether such a theory can support as well the description of stable systems or a system which makes a transition following the rule of Wigner and Weisskopf (as, for example, the adiabatic rotation of an atom with spin in an electromagnetic field). It is clear that if $D_{ \pm}$span the whole space, for example, there is no unstable subspace, and

$\ddagger$ It follows from (1.7) and the stability of $\mathcal{D}_{ \pm}$that $Z(\tau)=P_{\mathcal{K}} U(\tau) P_{\mathcal{K}}$ as well. 
one has a scattering theory without the type of resonances that can be associated with unstable systems. We shall treat this subject in more detail in a succeeding paper.

In the next section, we give a procedure for the construction of the subspaces $D_{ \pm}$, and for defining the representations which realize the Lax-Phillips structure. In this framework, we define the Lax-Phillips $S$-matrix. In Section 3, we show that this construction results in a Lax-Phillips theory applicable to models in which the underlying dynamics is locally defined in time. We carry out the construction for a Flesia-Piron type model. In Section 4 we study the general form of the Lax-Phillips $S$-matrix and prove that it is unitarily related to the standard $S$-matrix of the usual scattering theory in the auxiliary space. In Section 5, we work out the specific example of a time-independent Lee-Friedrichs spectral model $^{15}$, and show that the condition for the resonance eigenfunction is closely related to the resonance pole condition of the Lee-Friedrichs model of the usual quantum theory. A discussion and conclusions are given in Section 6 .

\section{The Subspaces $\mathcal{D}_{ \pm}$, Representations, and the Lax-Phillips $S$-Matrix}

It follows from the existence of the one-parameter unitary group $U(\tau)$ which acts on the Hilbert space $\overline{\mathcal{H}}$ that there is an operator $K$ which is the generator of dynamical evolution of the physical states in $\overline{\mathcal{H}}$; we assume that there exist wave operators $\Omega_{ \pm}$which intertwine this dynamical operator with an unperturbed dynamical operator $K_{0}$. We shall assume that $K_{0}$ has only absolutely continuous spectrum in $(-\infty, \infty)$.

We begin the development of the quantum Lax-Phillips theory with the construction of these representations. In this way, we shall construct explicitly the foliations described in Section 1. The free spectral representation of $K_{0}$ is defined by

$$
{ }_{f}\left\langle\sigma \beta\left|K_{0}\right| g\right\rangle=\sigma_{f}\langle\sigma \beta \mid g\rangle,
$$

where $|g\rangle$ is an element of $\overline{\mathcal{H}}$ and $\beta$ corresponds to the variables (measure space) of the auxiliary space associated to each value of $\sigma$, which, with $\sigma$, comprise a complete spectral set. The functions ${ }_{f}\langle\sigma \beta \mid g\rangle$ may be thought of as a set of functions of the variables $\beta$ indexed on the variable $\sigma$ in a continuous sequence of auxiliary Hilbert spaces isomorphic to $H$.

We now proceed to define the incoming and outgoing subspaces $\mathcal{D}_{ \pm}$. To do this, we define the Fourier transform from representations according to the spectrum $\sigma$ to the foliation variable $t$ of (1.5), i.e.,

$$
{ }_{f}\langle t \beta \mid g\rangle=\int e^{i \sigma t}{ }_{f}\langle\sigma \beta \mid g\rangle d \sigma
$$

Clearly, $K_{0}$ acts as the generator of translations in this representation. We shall say that the set of functions ${ }_{f}\langle t \beta \mid g\rangle$ are in the free translation representation.

Let us consider the sets of functions with support in $L^{2}(0, \infty)$ and in $L^{2}(-\infty, 0)$, and

call these subspaces $D_{0}^{ \pm}$. The Fourier transform back to the free spectral representation provides the two sets of Hardy class functions

$$
{ }_{f}\left\langle\sigma \beta \mid g_{0}^{ \pm}\right\rangle=\int e^{-i \sigma t}{ }_{f}\left\langle t \beta \mid g_{0}^{ \pm}\right\rangle d t \in H_{ \pm},
$$


for $g_{0}^{ \pm} \in D_{0}^{ \pm}$.

We may now define the subspaces $\mathcal{D}_{ \pm}$in the Hilbert space of states $\overline{\mathcal{H}}$. To do this we first map these Hardy class functions in $\bar{H}$ to $\overline{\mathcal{H}}$, i.e., we define the subspaces $\mathcal{D}_{0}^{ \pm}$by

$$
\int \sum_{\beta}|\sigma \beta\rangle_{f}{ }_{f}\left\langle\sigma \beta \mid g_{0}^{ \pm}\right\rangle d \sigma \in \mathcal{D}_{0}^{ \pm} .
$$

We shall assume that there are wave operators which intertwine $K_{0}$ with the full evolution $K$, i.e., that the limits

$$
\lim _{\tau \rightarrow \pm \infty} e^{i K \tau} e^{-i K_{0} \tau}=\Omega_{ \pm}
$$

exist on a dense set in $\overline{\mathcal{H}} . \S$

The construction of $\mathcal{D}_{ \pm}$is then completed with the help of the wave operators. We define these subspaces by

$$
\begin{aligned}
& \mathcal{D}_{+}=\Omega_{+} \mathcal{D}_{0}^{+} \\
& \mathcal{D}_{-}=\Omega_{-} \mathcal{D}_{0}^{-} .
\end{aligned}
$$

We remark that these subspaces are not produced by the same unitary map. This procedure is necessary to realize the Lax-Phillips structure non-trivially; if a single unitary map were used, then there would exist a transformation into the space of functions on $L^{2}(-\infty, \infty, H)$ which has the property that all functions with support on the positive half-line represent elements of $\mathcal{D}_{+}$, and all functions with support on the negative half-line represent elements of $\mathcal{D}_{-}$in the same representation; the resulting Lax-Phillips $S$-matrix would then be trivial. The requirement that $\mathcal{D}_{+}$and $\mathcal{D}_{-}$be orthogonal is not an immediate consequence of our construction; as we shall see, this result is associated with the analyticity of the operator which corresponds to the Lax-Phillips $S$-matrix.

In the following, we construct the Lax-Phillips $S$-matrix and the Lax-Phillips wave operators.

The wave operators defined by (2.5) intertwine $K$ and $K_{0}$, i.e.,

$$
K \Omega_{ \pm}=\Omega_{ \pm} K_{0}
$$

$\S$ We emphasize that the operator $K$ generates evolution of the entire virtual history, i.e., of elements in $\overline{\mathcal{H}}$, and that these wave operators are defined in this larger space. These operators are not,in general, the usual wave (intertwining) operators for the perturbed and unperturbed Hamiltonians that act in the auxiliary space. The conditions for their existence are, however, closely related to those of the usual wave operators. For the existence of the limit, it is sufficient that for $\tau \rightarrow \pm \infty,\left\|V e^{-i K_{0} \tau} \phi\right\| \rightarrow 0$ for a dense set in $\overline{\mathcal{H}}$. The free evolution may induce a motion of the wave packet in the auxiliary space out of the range of the potential (in the variables of the auxiliary space in the model representation), as for the usual scattering theory, so that it is possible to construct examples for which the wave operator exists if the potential falls off sufficiently rapidly. 
we may therefore construct the outgoing (incoming) spectral representations from the free spectral representation. Since

$$
\begin{aligned}
K \Omega_{ \pm}|\sigma \beta\rangle_{f} & =\Omega_{ \pm} K_{0}|\sigma \beta\rangle_{f} \\
& =\sigma \Omega_{ \pm}|\sigma \beta\rangle_{f}
\end{aligned}
$$

we may identify

$$
|\sigma \beta\rangle_{\substack{\text { out } \\ \text { in }}}=\Omega_{ \pm}|\sigma \beta\rangle_{f}
$$

The Lax-Phillips $S$-matrix is defined as the operator, on $\bar{H}$, which carries the incoming to outgoing translation representations of the evolution operator $K$. Suppose $g$ is an element of $\overline{\mathcal{H}}$; its incoming spectral representation, according to $(2.9)$, is

$$
\left.\left.{ }_{i n}\langle\sigma \beta| g\right)={ }_{f}\langle\sigma \beta| \Omega_{-}^{-1} g\right) .
$$

Let us now act on this function with the Lax-Phillips $S$-matrix in the free spectral representation, and require the result to be the outgoing representer of $g$ :

$$
\begin{aligned}
\left.{ }_{\text {out }}\langle\sigma \beta| g\right) & \left.={ }_{f}\langle\sigma \beta| \Omega_{+}^{-1} g\right) \\
& \left.=\int d \sigma^{\prime} \sum_{\beta^{\prime}}{ }_{f}\left\langle\sigma \beta|\mathbf{S}| \sigma^{\prime} \beta^{\prime}\right\rangle_{f} f\left\langle\sigma^{\prime} \beta^{\prime}\right| \Omega_{-}^{-1} g\right)
\end{aligned}
$$

where $\mathbf{S}$ is the Lax-Phillips $S$-operator (defined on $\overline{\mathcal{H}}$ ). Transforming the kernel to the free translation representation with the help of (2.2), i.e.,

$$
{ }_{f}\left\langle t \beta|\mathbf{S}| t^{\prime} \beta^{\prime}\right\rangle_{f}=\frac{1}{(2 \pi)^{2}} \int d \sigma d \sigma^{\prime} e^{i \sigma t} e^{-i \sigma^{\prime} t^{\prime}}\left\langle\sigma \beta|\mathbf{S}| \sigma^{\prime} \beta^{\prime}\right\rangle_{f}
$$

we see that the relation (2.11) becomes, after using Fourier transform in a similar way to transform the in and out spectral representations to the corresponding in and out translation representations,

$$
\begin{aligned}
\left.\left.{ }_{\text {out }}\langle t \beta| g\right)={ }_{f}\langle t \beta| \Omega_{+}^{-1} g\right) & \left.=\int d t^{\prime} \sum_{\beta^{\prime}}\left\langle t \beta|\mathbf{S}| t^{\prime} \beta^{\prime}\right\rangle_{f f}\left\langle t^{\prime} \beta^{\prime}\right| \Omega_{-}^{-1} g\right) \\
& \left.=\int d t^{\prime} \sum_{\beta^{\prime}}\left\langle t \beta|\mathbf{S}| t^{\prime} \beta^{\prime}\right\rangle_{f i n}\left\langle t^{\prime} \beta^{\prime}\right| g\right) .
\end{aligned}
$$

Hence the Lax-Phillips $S$-matrix is given by

$$
S=\left\{{ }_{f}\left\langle t \beta|\mathbf{S}| t^{\prime} \beta^{\prime}\right\rangle_{f}\right\}
$$

in free translation representation. It follows from the intertwining property (2.7) that

$$
{ }_{f}\left\langle\sigma \beta|\mathbf{S}| \sigma^{\prime} \beta^{\prime}\right\rangle_{f}=\delta\left(\sigma-\sigma^{\prime}\right) S^{\beta \beta^{\prime}}(\sigma)
$$


This result can be expressed in terms of operators on $\overline{\mathcal{H}}$. Let

$$
w_{-}^{-1}=\left\{{ }_{f}\langle t \beta| \Omega_{-}^{-1}\right\}
$$

be a map from $\overline{\mathcal{H}}$ to $\bar{H}$ in the incoming translation representation, and, similarly,

$$
w_{+}^{-1}=\left\{{ }_{f}\langle t \beta| \Omega_{+}^{-1}\right\}
$$

a map from $\overline{\mathcal{H}}$ to $\bar{H}$ in the outgoing translation representation. It then follows from (2.13) that

$$
S=w_{+}^{-1} w_{-},
$$

as a kernel on the free translation representation. This kernel is understood to operate on the representer of a vector $g$ in the incoming representation and map it to the representer in the outgoing representation.

We now discuss a class of pointwise physical models, and return in Section 4 to the construction of the Lax-Phillips $S$-matrix for this class of models.

\section{Pointwise Physical Models}

It has been shown by Piron $^{5}$ that if $\dagger K,-i \partial_{t}$, and $K+i \partial_{t}$ have a common dense domain on which they are essentially self-adjoint, then there exists an operator $\mathrm{H}$, defined as the self-adjoint extension of $K+i \partial_{t}$, which is a decomposable operator on $\bar{H}$, that is, $(\mathrm{H} \psi)_{t}=\mathrm{H}_{t} \psi_{t}$. We therefore have, on this common domain,

$$
K=-i \partial_{t}+\mathrm{H},
$$

corresponding to an evolution which acts pointwise in $t$. We shall identify the representation in which this analysis is carried out with what we have called the model representation.

In this section, we show that physical models of this type, for which the evolution is defined pointwise in time (in the model representation), which provide a straightforward way of lifting problems in the framework of the usual quantum theory to the Lax-Phillips structure, satisfy the requirements imposed by Eisenberg and Horwitz ${ }^{4}$ on the structure of a nontrivial Lax-Phillips theory, i.e., that the evolution be represented by a nontrivial kernel in the free translation representation.

Consider a class of models for nonrelativistic quantum theory characterized by the standard Heisenberg equations*

$$
\frac{d \mathbf{x}}{d t}=i[\mathrm{H}, \mathbf{x}] \quad \frac{d \mathbf{p}}{d t}=i[\mathrm{H}, \mathbf{p}]
$$

$\dagger$ The symbol $-i \partial_{t}$ stands, in this context, for the operator on $\overline{\mathcal{H}}$ which acts on the family $\left\{\mathcal{H}_{t}\right\}$ as a partial derivative in the foliation parameter.

* Context should avoid confusion between the symbol $\mathrm{H}$ for the Hamiltonian and the designation of the auxiliary Hilbert space $H$. 
in terms of operators defined on a Hilbert space $H$, where

$$
\mathrm{H}=\mathrm{H}_{0}+V \text {. }
$$

In case there is an explicit time dependence in $V=V(t)$, for example, in a model in which the interaction that induces instability is turned on at some finite laboratory time, it is often convenient to formally adjoin two new dynamical variables (as done, for example, by Piron ${ }^{5}$ and Howland $\left.{ }^{16}\right), T_{m}$ and $E$, along with an evolution parameter $\tau$ to replace the role of the parameter $t$ in (3.2) $\left(T_{m}\right.$ denotes the time operator in the space in which we construct the dynamical model of the system; such a time operator exists because the spectrum of $E$ is taken to be $(-\infty, \infty))$. The evolution operator may then be considered "time" $(\tau)$-independent, i.e., we define, as operators on a larger space $\overline{\mathcal{H}}$ (and thus identify $\mathrm{H}$ with the decomposable operator in (3.1)

$$
K=E+\mathrm{H}=K_{0}+V
$$

where

$$
K_{0}=E+\mathrm{H}_{0}
$$

and

$$
\left[T_{m}, E\right]=i
$$

Then, Eqs.(3.2) become

$$
\begin{aligned}
& \frac{d \mathbf{x}}{d \tau}=i[K, \mathbf{x}]=i[\mathrm{H}, \mathbf{x}] \\
& \frac{d \mathbf{p}}{d \tau}=i[K, \mathbf{p}]=i[\mathrm{H}, \mathbf{p}]
\end{aligned}
$$

and

$$
\begin{aligned}
\frac{d E}{d \tau} & =i[K, E]=i[\mathrm{H}, E] \\
\frac{d T_{m}}{d \tau} & =i\left[K, T_{m}\right]=i\left[E, T_{m}\right]=1 .
\end{aligned}
$$

The first of (3.8) implies, since $\mathrm{H}_{0}$ is independent of $t$, that ${ }^{16}$

$$
\frac{d E}{d \tau}=-\frac{\partial V}{\partial t}
$$

and the last of (3.8) puts $t$ and $\tau$ into correspondence, i.e., the expectation value of $t$ goes with $\tau$. The evolution of the system is, however, generated by the operator

$$
U(\tau)=e^{-i K \tau}
$$

corresponding to the Lax-Phillips evolution assumed in (1.3). The extension we have constructed (by the inclusion of the operators $T_{m}$ and $E$ ) enables us to embed the timedependent non-relativistic Heisenberg equations into the Lax-Phillips theory, in a way equivalent to the Flesia-Piron direct integral. The conditions that they impose, that $E$ 
and $K$ have a common dense domain, results, by means of the Trotter formula, in the conclusion that $\mathrm{H}$ acts pointwise in the spectral decompositon of $T_{m}$. This result gives (3.4) a precise meaning. That $K_{0}$ shares this common domain follows from the requirement that $V$ be "small" 17.

We shall label the spectral representation of the operator $T_{m}$ by the subscript $m$, so that for $\psi \in \overline{\mathcal{H}}$,

$$
\left.{ }_{m}\left\langle t \alpha\left|K_{0}\right| \psi\right)=-i \partial_{t m}\langle t \alpha| \psi\right)+{ }_{m}\left\langle t \alpha\left|\mathrm{H}_{0}\right| \psi\right),
$$

where $\{\alpha\}$ corresponds to a complete set in the (auxiliary) Hilbert space associated to $t$. We shall assume that $\mathrm{H}_{0}$ has no $t$ dependence, and that $V$ is diagonal in $t$, so that

$$
\left.{ }_{m}\left\langle t \alpha\left|\mathrm{H}_{0}\right| \psi\right)=\sum_{\alpha^{\prime}} \mathrm{H}_{0}^{\alpha, \alpha^{\prime}}{ }_{m}\left\langle t \alpha^{\prime}\right| \psi\right)
$$

and

$$
\left.{ }_{m}\langle t \alpha|V| \psi)=\sum_{\alpha^{\prime}} V^{\alpha, \alpha^{\prime}}(t)_{m}\left\langle t \alpha^{\prime}\right| \psi\right)
$$

We therefore see explicitly that the Hilbert space associated to the action of the operator $\mathrm{H}$ may be identified with the auxiliary space of the Lax-Phillips theory, and the larger space, representing the action of $T_{m}$ and $E$, with the function space $\bar{H}$ or the abstract space $\overline{\mathcal{H}}$ of the Lax-Phillips theory, as in the (direct integral) construction of Flesia and Piron.

The free spectral representation discussed in Section 2 is constructed by requiring that $K_{0}$, in this representation, act as multiplication. As in (2.1), we label this representation with subscript $f$, and require, for $\psi \in \overline{\mathcal{H}}$,

$$
\left.{ }_{f}\left\langle\sigma \beta\left|K_{0}\right| \psi\right)=\sigma_{f}\langle\sigma \beta| \psi\right),
$$

where $\{\beta\}$ corresponds to a complete set in the (auxiliary) Hilbert space associated to $\sigma$. This relation defines the free spectral representation.

The free translation representation is then given by (2.2), i.e.,

$$
\left.\left.{ }_{f}\langle t \beta| \psi\right)=\int_{-\infty}^{\infty} e^{i \sigma t}{ }_{f}\langle\sigma \beta| \psi\right) d \sigma
$$

One obtains, from (3.11)-(3.14), the relation

$$
\begin{aligned}
{ }_{m}\left\langle t \alpha\left|K_{0}\right| \sigma \beta\right\rangle_{f} & =\sigma_{m}\langle t \alpha \mid \sigma \beta\rangle_{f} \\
& =-i \partial_{t m}\langle t \alpha \mid \sigma \beta\rangle_{f}+\sum_{\alpha^{\prime}} H_{0}^{\alpha \alpha^{\prime}}{ }_{m}\left\langle t \alpha^{\prime} \mid \sigma \beta\right\rangle_{f} .
\end{aligned}
$$

Making the transformation

$$
{ }_{m}\langle t \alpha \mid \sigma \beta\rangle_{f}=e^{i \sigma t{ }_{m}^{0}\langle t \alpha \mid \sigma \beta\rangle_{f},}
$$


the relation $(3.16)$ becomes

$$
i \partial_{t}{ }_{m}^{0}\langle t \alpha \mid \sigma \beta\rangle_{f}=\sum_{\alpha^{\prime}} \mathrm{H}_{0}^{\alpha \alpha^{\prime}}{ }_{m}^{0}\left\langle t \alpha^{\prime} \mid \sigma \beta\right\rangle_{f}
$$

or

$$
{ }_{m}^{0}\langle t \alpha \mid \sigma \beta\rangle_{f}=\sum_{\alpha^{\prime}}\left(e^{-i \mathrm{H}_{0} t}\right)^{\alpha \alpha^{\prime}}{ }_{m}^{0}\left\langle 0 \alpha^{\prime} \mid \sigma \beta\right\rangle_{f} .
$$

The solution (3.19) of (3.18) is norm-preserving in $H$, and therefore ${ }_{m}^{0}\langle t \alpha \mid \sigma \beta\rangle_{f}$ are not elements of $\bar{H}$ (the integral of the modulus squared over $t$ diverges). This norm-preserving evolution reflects the stability of the system under evolution induced by $\mathrm{H}_{0}$. The factor $e^{i \sigma t}$ in (3.17) imbeds physical states into $\bar{H}$. To see this, consider the norm of $\left.{ }_{m}\langle t \alpha| \psi\right)$,

$$
\begin{aligned}
\left.\int d t \sum_{\alpha}\left|{ }_{m}\langle t \alpha| \psi\right)\right|^{2}= & \int d \sigma d \sigma^{\prime} d t \sum_{\alpha \beta \beta^{\prime}} e^{-i\left(\sigma-\sigma^{\prime}\right) t}{ }_{m}\langle t \alpha \mid \sigma \beta\rangle_{f}^{*} . \\
& \left.\left.\cdot{ }_{m}^{0}\left\langle t \alpha \mid \sigma^{\prime} \beta^{\prime}\right\rangle_{f}{ }_{f}\langle\sigma \beta| \psi\right)^{*}{ }_{f}\left\langle\sigma^{\prime} \beta^{\prime}\right| \psi\right) \\
= & \int d t d \sigma d \sigma^{\prime} \sum_{\alpha \ldots \beta^{\prime}} e^{-i\left(\sigma-\sigma^{\prime}\right) t}\left(e^{-i \mathrm{H}_{0} t}\right)^{\alpha \alpha^{\prime} *}\left(e^{-i \mathrm{H}_{0} t}\right)^{\alpha \alpha^{\prime \prime}} . \\
& \left.\left.\cdot{ }_{m}^{0}\left\langle 0 \alpha^{\prime} \mid \sigma \beta\right\rangle_{f}^{*}{ }_{m}^{0}\left\langle 0 \alpha^{\prime \prime} \mid \sigma^{\prime} \beta^{\prime}\right\rangle_{f}\langle\sigma \beta| \psi\right)^{*}{ }_{f}\left\langle\sigma^{\prime} \beta^{\prime}\right| \psi\right) .
\end{aligned}
$$

Carrying out the sum over $\alpha$, the unitary factors cancel, leaving $\delta_{\alpha^{\prime}, \alpha^{\prime \prime}}$. The $t$-integration then forms a factor $2 \pi \delta\left(\sigma-\sigma^{\prime}\right)$, permitting a sum on $\alpha^{\prime}=\alpha^{\prime \prime}$. We show below that, from the unitarity of ${ }_{f}\langle t \alpha \mid \sigma \beta\rangle_{f}$, it follows that the indices in ${ }_{m}\langle 0 \alpha \mid \sigma \beta\rangle_{f}$ label orthonormal sets in the auxiliary spaces attached to $t=0$ and $\sigma$, for each $\sigma$, i.e.,

$$
\sum_{\alpha}{ }_{m}^{0}\left\langle 0 \alpha^{\prime} \mid \sigma \beta\right\rangle_{f}^{*}{ }_{m}^{0}\left\langle 0 \alpha^{\prime} \mid \sigma \beta^{\prime}\right\rangle_{f}=\delta_{\beta, \beta^{\prime}}
$$

and the therefore the final integral on $\sigma$ and sum on $\beta$ can be carried out in (3.20):

$$
\left.\int d \sigma \sum_{\beta}\left|{ }_{f}\langle\sigma \beta| \psi\right)\right|^{2}=1
$$

On the other hand, if (3.19) were to provide the complete representation,

$$
\left.\left.\left.\sum_{\alpha, \alpha^{\prime}, \alpha^{\prime \prime}}\left(e^{-i \mathrm{H}_{0} t}\right)^{\alpha \alpha^{\prime} *}\left(e^{-i \mathrm{H}_{0} t}\right)^{\alpha \alpha^{\prime \prime}}{ }_{m}^{0}\left\langle 0 \alpha^{\prime}\right| \psi\right)^{*}{ }_{m}^{0}\left\langle 0 \alpha^{\prime \prime}\right| \psi\right)=\left.\sum_{\alpha^{\prime}}\right|_{m} ^{0}\left\langle 0 \alpha^{\prime}\right| \psi\right)\left.\right|^{2}
$$

is bounded but independent of $t$; an integral over $t$ would then diverge.

We now remark that since

$$
\left.{ }_{f}\left\langle\sigma \beta\left|e^{-i K_{0} \tau}\right| \psi\right)=e_{f}^{-i \sigma \tau}\langle\sigma \beta| \psi\right),
$$


it follows from (2.2) that

$$
\begin{aligned}
\left.{ }_{f}\langle t \beta| e^{-i K_{0} \tau} \psi\right) & \left.=\int d \sigma e^{i \sigma(t-\tau)}{ }_{f}\langle\sigma \beta| \psi\right) \\
& \left.={ }_{f}\langle t-\tau, \beta| \psi\right),
\end{aligned}
$$

making explicit the translation induced by $K_{0}$ in this representation, as is evident from (2.1) (or the first of (3.16)). It then follows that

$$
\left.{ }_{f}\left\langle t \beta\left|K_{0}\right| \psi\right)=-i \partial_{t f}\langle t \beta| \psi\right)
$$

and (3.16) becomes, in the free translation representation,

$$
\begin{aligned}
& { }_{m}\left\langle t \alpha\left|K_{0}\right| t^{\prime} \beta\right\rangle_{f}=i \partial_{t^{\prime} m}\left\langle t \alpha \mid t^{\prime} \beta\right\rangle_{f}
\end{aligned}
$$

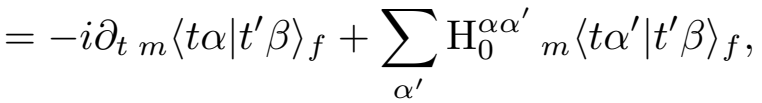

or

$$
\left(i \partial_{t}+\partial_{t^{\prime}}\right)_{m}\left\langle t \alpha \mid t^{\prime} \beta\right\rangle_{f}=\sum_{\alpha^{\prime}} \mathrm{H}_{0}^{\alpha \alpha^{\prime}}{ }_{m}\left\langle t \alpha^{\prime} \mid t^{\prime} \beta\right\rangle_{f}
$$

It is clear from (3.26) that the transformation function, ${ }_{m}\left\langle t \alpha \mid t^{\prime} \beta\right\rangle_{f}$, from the representation in which $T_{m}$ is diagonal,

$$
T_{m}=\int d t \sum_{\alpha}|t \alpha\rangle_{m} t_{m}\langle t \alpha|,
$$

to that for which the free time operator

$$
T_{f}=\int d t \sum_{\beta}|t \beta\rangle_{f} t_{f}\langle t \beta|
$$

is diagonal, cannot be a function of $t-t^{\prime}$ alone (in particular, proportional to $\delta\left(t-t^{\prime}\right)$ ), if the right hand side of (3.26) is not zero. We see that the existence of a free Schrödinger type evolution operator, which can propagate a stable state, is necessary for the non-trivially different structure of the free and model translation representations.

To find the general solution of (3.26), let

$$
{ }_{m}\left\langle t \alpha \mid t^{\prime} \beta\right\rangle_{f}=f^{\alpha \beta}\left(t_{+}, t_{-}\right),
$$

where

$$
t_{ \pm}=\frac{t^{\prime} \pm t}{2}
$$

Then, (3.26) becomes

$$
i \partial_{t_{+}} f^{\alpha \beta}\left(t_{+}, t_{-}\right)=\sum_{\alpha^{\prime}} \mathrm{H}_{0}^{\alpha \alpha^{\prime}} f^{\alpha^{\prime} \beta}\left(t_{+}, t_{-}\right)
$$


with solution

$$
f^{\alpha \beta}\left(t_{+}, t_{-}\right)=\sum_{\alpha^{\prime}}\left(e^{-i \mathrm{H}_{0} t_{+}}\right)^{\alpha \alpha^{\prime}} f^{\alpha^{\prime} \beta}\left(0, t_{-}\right) .
$$

It therefore follows that

$$
\begin{aligned}
{ }_{m}\langle t \alpha \mid \sigma \beta\rangle_{f} & =\sum_{\alpha^{\prime}} \int d t^{\prime} e^{i \sigma t^{\prime}}\left(e^{-i \mathrm{H}_{0} t_{+}}\right)^{\alpha \alpha^{\prime}} f^{\alpha^{\prime} \beta}\left(0, t_{-}\right) \\
& =\sum_{\alpha^{\prime} \alpha^{\prime \prime}} \int d t^{\prime} e^{i \sigma t^{\prime}}\left(e^{-i \mathrm{H}_{0} t}\right)^{\alpha \alpha^{\prime \prime}}\left(e^{-i \mathrm{H}_{0} \frac{t^{\prime}-t}{2}}\right)^{\alpha^{\prime \prime} \alpha^{\prime}} f^{\alpha^{\prime} \beta}\left(0, t_{-}\right) \\
& =\sum_{\alpha^{\prime} \alpha^{\prime \prime}} \int d\left(t^{\prime}-t\right) e^{i \sigma t} e^{i \sigma\left(t^{\prime}-t\right)}\left(e^{-i \mathrm{H}_{0} t}\right)^{\alpha \alpha^{\prime \prime}}\left(e^{-i \mathrm{H}_{0} \frac{t^{\prime}-t}{2}}\right)^{\alpha^{\prime \prime} \alpha^{\prime}} f^{\alpha^{\prime} \beta}\left(0, t_{-}\right) .
\end{aligned}
$$

We now define

$$
U^{\alpha \beta}(\sigma)=\sqrt{2 \pi} \int d t e^{i \sigma t}\left(e^{-i \mathrm{H}_{0} t / 2}\right)^{\alpha \alpha^{\prime}} f^{\alpha^{\prime} \beta}(0, t / 2)
$$

so that (3.32) becomes

$$
{ }_{m}\langle t \alpha \mid \sigma \beta\rangle_{f}=\frac{1}{\sqrt{2 \pi}} \sum_{\alpha^{\prime}} e^{i \sigma t}\left(e^{-i \mathrm{H}_{0} t}\right)^{\alpha \alpha^{\prime}} U^{\alpha^{\prime} \beta}(\sigma) .
$$

It then follows that

$$
U^{\alpha \beta}(\sigma)=\sqrt{2 \pi}_{m}\langle 0 \alpha \mid \sigma \beta\rangle_{f} .
$$

The unitarity relations for the transformation function ${ }_{m}\langle t \alpha \mid \sigma \beta\rangle_{f}$ imply the unitarity of $U^{\alpha \beta}(\sigma)$ :

$$
\begin{aligned}
\sum_{\alpha} \int d t_{f}\langle\sigma \beta \mid t \alpha\rangle_{m m}\left\langle t \alpha \mid \sigma^{\prime} \beta^{\prime}\right\rangle_{f}= & \frac{1}{2 \pi} \sum_{\alpha \alpha^{\prime} \alpha^{\prime \prime}} \int d t e^{-i \sigma t}\left(e^{-i \mathrm{H}_{0} t}\right)^{\alpha \alpha^{\prime} *} U^{\alpha^{\prime} \beta *}(\sigma) . \\
& \cdot e^{i \sigma^{\prime} t}\left(e^{-i \mathrm{H}_{0} t}\right)^{\alpha \alpha^{\prime \prime}} U_{\alpha^{\prime \prime} \beta^{\prime}} \\
= & \delta\left(\sigma-\sigma^{\prime}\right) \sum_{\alpha} U^{\alpha \beta *}(\sigma) U^{\alpha \beta^{\prime}}(\sigma)
\end{aligned}
$$

so that

$$
\sum_{\alpha} U^{\alpha \beta *}(\sigma) U^{\alpha \beta^{\prime}}(\sigma)=\delta_{\beta \beta^{\prime}}
$$

Moreover,

$$
\begin{aligned}
& \sum_{\beta} \int d \sigma_{m}\langle t \alpha \mid \sigma \beta\rangle_{f f}\left\langle\sigma \beta \mid t^{\prime} \alpha^{\prime}\right\rangle_{m}= \\
& \quad=\frac{1}{2 \pi} \sum_{\beta \alpha^{\prime \prime} \alpha^{\prime \prime \prime}} \int d \sigma e^{i \sigma\left(t-t^{\prime}\right)}\left(e^{-i \mathrm{H}_{0} t}\right)^{\alpha \alpha^{\prime \prime}}\left(e^{-i \mathrm{H}_{0} t^{\prime}}\right)^{\alpha^{\prime} \alpha^{\prime \prime \prime} *} \cdot U^{\alpha^{\prime \prime} \beta}(\sigma) U^{\alpha^{\prime \prime \prime} \beta *}(\sigma) \\
& \quad=\delta\left(t-t^{\prime}\right) \delta_{\alpha \alpha^{\prime}} .
\end{aligned}
$$


Now, suppose that $\alpha, \alpha^{\prime}$ correspond to (generalized) eigenstates of $\mathrm{H}_{0}$; then, (3.37) becomes

$$
\delta\left(t-t^{\prime}\right) \delta_{\alpha \alpha^{\prime}}=\frac{1}{2 \pi} \sum_{\beta} \int d \sigma e^{i\left(\sigma-E_{\alpha}\right) t} e^{-i\left(\sigma-E_{\alpha^{\prime}}\right) t^{\prime}} U^{\alpha \beta}(\sigma) U^{\alpha^{\prime} \beta *}(\sigma)
$$

Multiplying (3.38) by $e^{-i \nu t}$ and integrating over $t$, we obtain

$$
e^{-i \nu t^{\prime}} \delta_{\alpha \alpha^{\prime}}=\left.e^{-i\left(\nu+E_{\alpha}-E_{\alpha^{\prime}}\right) t^{\prime}} \sum_{\beta} U^{\alpha \beta}(\sigma) U^{\alpha^{\prime} \beta *}(\sigma)\right|_{\sigma=E_{\alpha}+\nu}
$$

for every $\nu$. This relation implies that $E_{\alpha}=E_{\alpha^{\prime}}$, so that

$$
\delta_{\alpha \alpha^{\prime}}=\sum_{\beta} U^{\alpha \beta}(\sigma) U^{\alpha^{\prime} \beta *}(\sigma) .
$$

The transformation function ${ }_{m}\langle t \alpha \mid \sigma \beta\rangle_{f}=e^{i \sigma t{ }_{m}}\langle t \alpha \mid \sigma \beta\rangle_{f}$ constitutes a map from the spectral family associated with $T_{m}$, represented by the kets $\left\{|t \alpha\rangle_{m}\right\}$ to the spectral representation of $K_{0}$, represented by the kets $\left\{|\sigma \beta\rangle_{f}\right\}$. We can think of this map in two stages, the first from $\left\{|t \alpha\rangle_{m}\right\}$ to a standard frame $\left\{\left|\beta^{\prime}\right\rangle_{0}\right\}$ (projection) in the auxiliary space of the free representation, then a map (lift) from this to the foliated frames $\left\{|\sigma \beta\rangle_{f}\right\}$ according to

$$
{ }_{m}\langle t \alpha \mid \sigma \beta\rangle_{f}=\sum_{\beta^{\prime}}{ }_{m}\left\langle t \alpha \mid \beta^{\prime}\right\rangle_{0}{ }_{0}\left\langle\beta^{\prime} \mid \sigma \beta\right\rangle_{f}
$$

with the property (3.17) due to the contraction with ${ }_{0}\left\langle\beta^{\prime} \mid \sigma \beta\right\rangle_{f}$. Then, (3.35) can be written as

$$
U^{\alpha \beta}(\sigma)=\sqrt{2 \pi} \sum_{\beta^{\prime}} m\left\langle 0 \alpha \mid \beta^{\prime}\right\rangle_{0}{ }_{0}\left\langle\beta^{\prime} \mid \sigma \beta\right\rangle_{f} .
$$

Let us define the unitary map

$$
\left\langle\alpha \mid \beta^{\prime}\right\rangle \equiv \sqrt{2 \pi}_{m}\left\langle 0 \alpha \mid \beta^{\prime}\right\rangle_{0}
$$

so that

$$
\begin{aligned}
U^{\beta^{\prime} \beta}(\sigma) & \equiv{ }_{0}\left\langle\beta^{\prime} \mid \sigma \beta\right\rangle_{f} \\
& =\sum_{\alpha}\left\langle\beta^{\prime} \mid \alpha\right\rangle U^{\alpha \beta}(\sigma)
\end{aligned}
$$

corresponds to a transformation in "orientation" of the representation from the standard one, in the isomorphic auxiliary spaces. The map $U^{\beta^{\prime} \beta}(\sigma)$ from a standard frame to a frame varying with $\sigma$ has the geometric interpretation of a section of a frame bundle, as reflected in (3.40).

\section{The S-Matrix for Pointwise Models.}

In this section we define the Lax-Phillips wave operators for the pointwise models discussed in the previous section, and compute the $S$-matrix (based on the intertwining 
of $K$ and $K_{0}$ ). We show that the Lax-Phillips $S$-matrix is, in this case, simply related to the $S$-matrix of the usual scattering problem (based on the intertwining of $\mathrm{H}$ and $\mathrm{H}_{0}$ ) by the unitary operator $U(\sigma)$. This operator acts in a way similar to that of the dilation used by Aguilar and Combes ${ }^{18}$ (see also Simon ${ }^{19}$ ); analytic continuation in $\sigma$ distorts the continuous spectrum of the Hamiltonian, exposing the resonance poles on the first sheet. We give an example, based on the Lee-Friedrichs model ${ }^{15}$ in the next section.

We show in the following that the spectrally diagonal operator $S^{\beta \beta^{\prime}}(\sigma)$ for pointwise models has the form

$$
S^{\beta \beta^{\prime}}(\sigma)=U^{\alpha \beta *}(\sigma)\left(S^{a u x}\right)^{\alpha \alpha^{\prime}} U^{\alpha^{\prime} \beta^{\prime}}(\sigma) .
$$

Here, $U^{\alpha \beta}(\sigma)$ is the operator on the auxiliary space defined by (3.35), and $S^{\text {aux }}$ is the $S$-matrix of the usual scattering theory defined by $\mathrm{H}, \mathrm{H}_{0}$ in the auxiliary space.

To see this, we study the operator $\mathbf{S}$ in the form

$$
\mathbf{S}=\Omega_{+}^{-1} \Omega_{-}=\lim _{\tau \rightarrow \infty} e^{i K_{0} \tau} e^{-2 i K \tau} e^{i K_{0} \tau},
$$

which can be expressed as

$$
\begin{aligned}
\mathbf{S}= & \lim _{\epsilon \rightarrow 0} \epsilon \int_{0}^{\infty} d \tau e^{-\epsilon \tau} e^{i K_{0} \tau} e^{-2 i K \tau} e^{i K_{0} \tau} \\
= & \int_{0}^{\infty} d \tau\left(-\frac{d}{d \tau} e^{-\epsilon \tau}\right) e^{i K_{0} \tau} e^{-2 i K \tau} e^{i K_{0} \tau} \\
= & 1-i \int_{0}^{\infty} d \tau\left\{e^{i K_{0} \tau} V e^{-2 i K \tau} e^{i K_{0} \tau}\right. \\
& \left.+e^{i K_{0} \tau} e^{-2 i K \tau} V e^{i K_{0} \tau}\right\} e^{-\epsilon \tau}
\end{aligned}
$$

In the free spectral representation, we therefore have

$$
\begin{aligned}
{ }_{f}\left\langle\sigma \beta|\mathbf{S}| \sigma^{\prime} \beta^{\prime}\right\rangle_{f} & =\delta\left(\sigma-\sigma^{\prime}\right) \delta^{\beta \beta^{\prime}} \\
& -i \int_{0}^{\infty} d \tau_{f}\left\langle\sigma \beta\left|V e^{i\left(\sigma+\sigma^{\prime}-2 K+i \epsilon\right) \tau}+e^{i\left(\sigma+\sigma^{\prime}-2 K+i \epsilon\right) \tau} V\right| \sigma^{\prime} \beta^{\prime}\right\rangle_{f} \\
& =\delta\left(\sigma-\sigma^{\prime}\right) \delta^{\beta \beta^{\prime}} \\
& +\frac{1}{2}{ }_{f}\left\langle\sigma \beta\left|V G\left(\frac{\sigma+\sigma^{\prime}}{2}+i \epsilon\right)+G\left(\frac{\sigma+\sigma^{\prime}}{2}+i \epsilon\right) V\right| \sigma^{\prime} \beta^{\prime}\right\rangle_{f},
\end{aligned}
$$

where we use the definitions

$$
G(z)=\frac{1}{z-K}, \quad G_{0}(z)=\frac{1}{z-K_{0}} .
$$

We now define the operator ${ }^{20}$

$$
\mathbf{T}(z)=V+V G(z) V=V+V G_{0}(z) \mathbf{T}(z),
$$


where we have used the second resolvent equation

$$
\begin{aligned}
G(z) & =G_{0}(z)+G_{0}(z) V G(z) \\
& =G_{0}(z)+G(z) V G_{0}(z) .
\end{aligned}
$$

Since

$$
\begin{aligned}
\mathbf{T}(z) G_{0}(z) & =V G_{0}(z)+V G(z) V G_{0}(z) \\
& =V G(z),
\end{aligned}
$$

and

$$
\begin{aligned}
G_{0}(z) \mathbf{T}(z) & =G_{0}(z) V+G_{0}(z) V G(z) V \\
& =G(z) V
\end{aligned}
$$

it follows that

$$
\begin{aligned}
{ }_{f}\left\langle\sigma \beta|\mathbf{S}| \sigma^{\prime} \beta^{\prime}\right\rangle_{f}= & \delta\left(\sigma-\sigma^{\prime}\right) \delta^{\beta \beta^{\prime}} \\
+ & \frac{1}{2} f\langle\sigma \beta| \mathbf{T}\left(\frac{\sigma+\sigma^{\prime}}{2}+i \epsilon\right) G_{0}\left(\frac{\sigma+\sigma^{\prime}}{2}+i \epsilon\right) \\
& +G_{0}\left(\frac{\sigma+\sigma^{\prime}}{2}+i \epsilon\right) \mathbf{T}\left(\frac{\sigma+\sigma^{\prime}}{2}+i \epsilon\right)\left|\sigma^{\prime} \beta^{\prime}\right\rangle_{f} \\
= & \delta\left(\sigma-\sigma^{\prime}\right) \delta^{\beta \beta^{\prime}}+\left\{\frac{1}{\sigma-\sigma^{\prime}+i \epsilon}+\frac{1}{\sigma^{\prime}-\sigma+i \epsilon}\right\}_{f}\left\langle\sigma \beta\left|\mathbf{T}\left(\frac{\sigma+\sigma^{\prime}}{2}+i \epsilon\right)\right| \sigma^{\prime} \beta^{\prime}\right\rangle_{f} \\
= & \delta\left(\sigma-\sigma^{\prime}\right)\left\{\delta^{\beta \beta^{\prime}}-2 \pi i{ }_{f}\left\langle\sigma \beta|\mathbf{T}(\sigma+i \epsilon)| \sigma \beta^{\prime}\right\rangle_{f}\right\} .
\end{aligned}
$$

We remark that by this construction, we see that $S^{\beta \beta^{\prime}}(\sigma)$ is analytic in the upper half plane in $\sigma$.

To complete our demonstration of (4.1), we expand $\mathbf{T}(z)$ (aasuming that the series converges), using (4.6), as

$$
\mathbf{T}=V+V G_{0}(z) V+V G_{0}(z) V G_{0}(z) V+\cdots .
$$

The matrix elements of $\mathbf{T}$ therefore involve

$$
{ }_{f}\left\langle\sigma \beta|V| \sigma^{\prime} \beta^{\prime}\right\rangle_{f}=\int d t \sum_{\alpha \alpha^{\prime}}\langle\sigma \beta \mid t \alpha\rangle_{m} V_{m}^{\alpha \alpha^{\prime}}\left\langle t \alpha^{\prime} \mid \sigma^{\prime} \beta^{\prime}\right\rangle_{f} .
$$

From (3.34), we obtain

$$
{ }_{f}\left\langle\sigma \beta|V| \sigma^{\prime} \beta^{\prime}\right\rangle_{f}=\frac{1}{2 \pi} \sum_{\alpha \alpha^{\prime}} \int d t e^{i\left(\sigma^{\prime}-\sigma\right) t} U^{\alpha \beta *}(\sigma) V_{I}(t)^{\alpha \alpha^{\prime}} U^{\alpha^{\prime} \beta^{\prime}}\left(\sigma^{\prime}\right),
$$

where $V_{I}(t)$ is the interaction picture form for $V$ in the standard scattering theory,

$$
V_{I}^{\alpha \alpha^{\prime}}(t)=\sum_{\alpha^{\prime \prime} \alpha^{\prime \prime \prime}}\left(e^{i H_{0} t}\right)^{\alpha \alpha^{\prime \prime}} V^{\alpha^{\prime \prime} \alpha^{\prime \prime \prime}}(t)\left(e^{-i H_{0} t}\right)^{\alpha^{\prime \prime \prime} \alpha^{\prime}} .
$$


It is convenient to write (4.13) as an operator-valued kernel on the auxiliary space in the free spectral representation (suppressing the explicit indices of the auxiliary space), i.e.,

$$
{ }_{f}\left\langle\sigma|V| \sigma^{\prime}\right\rangle_{f}=\frac{1}{2 \pi} \int d t e^{i\left(\sigma^{\prime}-\sigma\right) t} U^{\dagger}(\sigma) V_{I}(t) U\left(\sigma^{\prime}\right) .
$$

Since

$$
{ }_{f}\left\langle\sigma^{\prime}\left|G_{0}(\sigma+i \epsilon)\right| \sigma^{\prime \prime}\right\rangle_{f}=\frac{1}{\sigma-\sigma^{\prime}+i \epsilon} \delta\left(\sigma^{\prime}-\sigma^{\prime \prime}\right)
$$

it follows that

$$
\begin{aligned}
& f_{f}\left\langle\sigma\left|V G_{0}(\sigma+i \epsilon) V\right| \sigma^{\prime}\right\rangle_{f}=\int d \sigma^{\prime \prime} d \sigma^{\prime \prime \prime} . \\
& \cdot{ }_{f}\left\langle\sigma|V| \sigma^{\prime \prime}\right\rangle_{f}\left\langle\sigma^{\prime \prime}\left|G_{0}(\sigma+i \epsilon)\right| \sigma^{\prime \prime \prime}\right\rangle_{f} f_{f}\left\langle\sigma^{\prime \prime \prime}|V| \sigma^{\prime}\right\rangle \\
&= U^{\dagger}(\sigma) \frac{1}{(2 \pi)^{2}} \int d \sigma^{\prime \prime} d t d t^{\prime} \frac{e^{i \sigma^{\prime \prime}\left(t-t^{\prime}\right)}}{\sigma-\sigma^{\prime \prime}+i \epsilon} e^{-i \sigma t} e^{i \sigma^{\prime} t^{\prime}} V_{I}(t) V_{I}\left(t^{\prime}\right) U(\sigma) .
\end{aligned}
$$

Closing the contour in the upper half plane in $\sigma^{\prime \prime}$ to include the pole at $\sigma^{\prime \prime}=\sigma+i \epsilon$ requires $t>t^{\prime}$ (for $t<t^{\prime}$, the contour must be closed in the lower half plane and vanishes); the result, for $t>t^{\prime}$, is $-2 \pi i e^{i(\sigma+i \epsilon)\left(t-t^{\prime}\right)}$, so that

$$
{ }_{f}\left\langle\sigma\left|V G_{0}(\sigma+i \epsilon) V\right| \sigma^{\prime}\right\rangle_{f}=-\frac{i}{2 \pi} U^{\dagger}(\sigma) \int_{-\infty}^{\infty} d t \int_{-\infty}^{t} d t^{\prime} e^{i\left(\sigma^{\prime}-\sigma\right) t} V_{I}(t) V_{I}\left(t^{\prime}\right) U\left(\sigma^{\prime}\right) .
$$

For $\sigma=\sigma^{\prime}$, as enforced by (4.10), the exponential factor is unity.

To see how the rest of the series goes, we calculate

$$
\begin{aligned}
{ }_{f}\left\langle\sigma\left|V G_{0}(\sigma+i \epsilon) V G_{0}(\sigma+i \epsilon) V\right| \sigma^{\prime}\right\rangle_{f}= \\
=\frac{1}{(2 \pi)^{3}} U^{\dagger}(\sigma) \int d t d t^{\prime} d t^{\prime \prime} d \sigma^{\prime \prime} d \sigma^{\prime \prime \prime} \frac{e^{i\left(\sigma^{\prime \prime}-\sigma\right) t} e^{i\left(\sigma^{\prime \prime \prime}-\sigma^{\prime \prime}\right) t^{\prime}} e^{i\left(\sigma^{\prime}-\sigma^{\prime \prime \prime}\right) t^{\prime \prime}}}{\left(\sigma-\sigma^{\prime \prime}+i \epsilon\right)\left(\sigma-\sigma^{\prime \prime \prime}+i \epsilon\right)} \\
\quad \cdot V_{I}(t) V_{I}\left(t^{\prime}\right) V_{I}\left(t^{\prime \prime}\right) U\left(\sigma^{\prime}\right)
\end{aligned}
$$

where the internal factors $U\left(\sigma^{\prime \prime}\right), U\left(\sigma^{\prime \prime \prime}\right)$ cancel. Now, as above,

$$
\int d \sigma^{\prime \prime} \frac{e^{i \sigma^{\prime \prime}\left(t-t^{\prime}\right)}}{\sigma-\sigma^{\prime \prime}+i \epsilon}=-2 \pi i e^{i \sigma\left(t-t^{\prime}\right)} t>t^{\prime}
$$

and is otherwise zero. The integral over $\sigma^{\prime \prime \prime}$ then yields

$$
\int d \sigma^{\prime \prime \prime} \frac{e^{i \sigma^{\prime \prime \prime}\left(t^{\prime}-t^{\prime \prime}\right)}}{\sigma-\sigma^{\prime \prime \prime}+i \epsilon}=-2 \pi i e^{i \sigma\left(t^{\prime}-t^{\prime \prime}\right)} t^{\prime}>t^{\prime \prime}
$$

and is otherwise zero, so we conclude that a non-zero result requires $t>t^{\prime}>t^{\prime \prime}$, and in this case

$$
\begin{aligned}
& f\left\langle\sigma\left|V G_{0}(\sigma+i \epsilon) V G_{0}(\sigma+i \epsilon) V\right| \sigma^{\prime}\right\rangle_{f}= \\
& \quad=\frac{i^{2}}{2 \pi} U^{\dagger}(\sigma) \int_{-\infty}^{\infty} d t \int_{\infty}^{t} d t^{\prime} \int_{-\infty}^{t^{\prime}} d t^{\prime \prime} V_{I}(t) V_{I}\left(t^{\prime}\right) V_{I}\left(t^{\prime \prime}\right) U\left(\sigma^{\prime}\right) e^{i\left(\sigma^{\prime}-\sigma\right) t^{\prime \prime}}
\end{aligned}
$$


the last factor again becomes unity under the restriction $\sigma=\sigma^{\prime}$. The general result for the series is

$$
\begin{aligned}
f\left\langle\sigma|\mathbf{S}| \sigma^{\prime}\right\rangle_{f} & =\delta\left(\sigma-\sigma^{\prime}\right) U^{\dagger}(\sigma)\left\{1-i \int_{-\infty}^{\infty} d t V_{I}(t)\right. \\
& +\frac{(-i)^{2}}{2 !} \mathcal{T} \int_{-\infty}^{\infty} d t d t^{\prime} V_{I}(t) V_{I}\left(t^{\prime}\right) \\
& +\frac{(-i)^{3}}{3 !} \mathcal{T} \int_{-\infty}^{\infty} d t d t^{\prime} d t^{\prime \prime} V_{I}(t) V_{I}\left(t^{\prime}\right) V_{I}\left(t^{\prime \prime \prime}\right) \\
& +\cdots\} U(\sigma)
\end{aligned}
$$

where $\mathcal{T}$ indicates that the operations must be time-ordered under the integrals. The terms in the bracket in (4.20) are the expansion of

$$
S^{a u x}=\mathcal{T}\left(e^{-i \int_{-\infty}^{\infty} V_{I}(t) d t}\right)
$$

so that (4.1) is proven.

We have constructed the incoming and outgoing subspaces $\mathcal{D}_{ \pm}$in (2.6). It is essential for application of the Lax-Phillips theory that these subspaces be orthogonal, i.e., for every $f_{+} \in \mathcal{D}_{+}, f_{-} \in \mathcal{D}_{-}$, that $\left(f_{+}, f_{-}\right)=0$. If

$$
\begin{aligned}
& f_{+}=\Omega_{+} f_{0}^{+} \\
& f_{-}=\Omega_{-} f_{0}^{-}
\end{aligned}
$$

mapped from functions in $\mathcal{D}_{0}^{ \pm}$, we see that the orthogonality condition is

$$
\left(f_{+}, f_{-}\right)=\left(f_{0}^{+}, \Omega_{+}^{-1} \Omega_{-} f_{0}^{-}\right)=0 .
$$

We now show that the $S$-matrix leaves the support of the functions in $\mathcal{D}_{-}$in the incoming representation invariant, ${ }^{1}$ and therefore the orthogonality condition is satisfied. As shown in (2.11), the $S$-matrix in free representation transforms the incoming to the outgoing representation; we may therefore write the scalar product in (4.23) as

$$
\left(f_{+}, f_{-}\right)=\sum_{\beta \beta^{\prime}} \int d t d t^{\prime}\left(f_{0}^{+}|t \beta\rangle_{\text {out } f}\left\langle t \beta|\mathbf{S}| t^{\prime} \beta^{\prime}\right\rangle_{f \text { in }}\left\langle t^{\prime} \beta^{\prime}\right| f_{0}^{-}\right)
$$

Now,

$$
\begin{aligned}
{ }_{f}\left\langle t \beta|\mathbf{S}| t^{\prime} \beta^{\prime}\right\rangle_{f} & =\int d \sigma d \sigma^{\prime} e^{i \sigma t} e^{-i \sigma^{\prime} t^{\prime}}\left\langle\sigma \beta|\mathbf{S}| \sigma^{\prime} \beta^{\prime}\right\rangle_{f} \\
& =\int d \sigma e^{i \sigma\left(t-t^{\prime}\right)} S^{\beta \beta^{\prime}}(\sigma) \\
& =S^{\beta \beta^{\prime}}\left(t-t^{\prime}\right) .
\end{aligned}
$$

The function $S(\sigma)^{\beta \beta^{\prime}}$ is analytic in the upper half plane; it may have a null co-space, but is otherwise regular. Its singularity lies in the lower half plane . To find the non-vanishing 
value for $S^{\beta \beta^{\prime}}\left(t-t^{\prime}\right)$, we must close the contour in the lower half plane. This can only be done if $t^{\prime}>t$. For $t^{\prime}<t$, one must close in the upper half plane, and there $S(\sigma)$ has no singularity, so the integral vanishes. Hence $S^{\beta \beta^{\prime}}\left(t-t^{\prime}\right)$ takes $\mathcal{D}_{-}$to $\mathcal{D}_{-}$in the incoming representation, and the subspaces $\mathcal{D}_{+}$and $\mathcal{D}_{-}$are orthogonal.

We finally remark that the $S$-matrix, in the model space, has the form

$$
\begin{aligned}
{ }_{m}\left\langle t \alpha|\mathbf{S}| t^{\prime} \alpha^{\prime}\right\rangle_{m}= & \sum_{\beta \beta^{\prime}} \int d \sigma d \sigma_{m}^{\prime}\langle t \alpha \mid \sigma \beta\rangle_{f}\left\langle\sigma \beta|\mathbf{S}| \sigma^{\prime} \beta^{\prime}\right\rangle_{f}{ }_{f}\left\langle\sigma^{\prime} \beta^{\prime} \mid t^{\prime} \alpha^{\prime}\right\rangle_{m} \\
= & \sum_{\beta \beta^{\prime}} \int d \sigma_{m}\langle t \alpha \mid \sigma \beta\rangle_{f} U^{\dagger \beta \alpha}(\sigma) S^{a u x, \alpha \alpha^{\prime}} U^{\alpha^{\prime} \beta^{\prime}}(\sigma)_{f}\left\langle\sigma \beta^{\prime} \mid t^{\prime} \alpha^{\prime}\right\rangle_{m} \\
= & \frac{1}{2 \pi} \int d \sigma e^{i \sigma\left(t-t^{\prime}\right)}\left(e^{-i H_{0} t}\right)^{\alpha \alpha^{\prime \prime}} U^{\alpha^{\prime \prime} \beta}(\sigma) U^{\dagger \beta \alpha^{\prime \prime \prime}}(\sigma) . \\
& \cdot S^{a u x, \alpha^{\prime \prime \prime} \alpha^{i v}} U^{\alpha^{i v} \beta^{\prime}}(\sigma) U^{\dagger \beta^{\prime} \alpha^{v}}(\sigma)\left(e^{-i H_{0} t^{\prime}}\right)^{\alpha^{v} \alpha^{\prime} *} \\
= & \delta\left(t-t^{\prime}\right) S^{a u x, \alpha \alpha^{\prime}},
\end{aligned}
$$

where we have used (3.34) and the fact that $H_{0}$ commutes with $S^{\text {aux }}$. In the model space, $S^{a u x}$ acts at a given $t$, and multiplication by $\delta\left(t-t^{\prime}\right)$ constitutes the lift of this operator to the Lax-Phillips theory. This result illustrates the conclusion of ref. 4, that for a Hamiltonian that is pointwise in $t$, the Lax-Phillips $S$-matrix has no non-trivial analytic structure in the model representation. In the free spectral representation, however, it has the non-trivial analytic structure necessary for establishing the relation between the singularities of $S(\sigma)$ and the spectrum of the generator of the semigroup.

\section{The Lee-Friedrichs Model}

In this section, we work out a specific illustrative example, the well-known time independent soluble model of Friedrichs and Lee ${ }^{15}$. We show that, for a simple choice of $U(\sigma)$, the exponential Lax-Phillips decay law can coincide with the Lee-Friedrichs pole approximation.

The Lee-Friedrichs model for scattering and resonances ${ }^{15,21}$, in the framework of standard non-relativistic scattering theory, is characterized by a Hamiltonian $\mathrm{H}=\mathrm{H}_{0}+V$ for which $\mathrm{H}_{0}$ has a bound state with eigenfunction $\phi$ and eigenvalue $E_{0}$ embedded in an absolutely continuous spectrum on $(0, \infty)$, and for which $V$ has matrix elements only from the discrete bound state to the generalized eigenfunctions on the continuum. The vanishing of continuum-continuum matrix elements corresponds to the assumption, often a good approximation, that there are no final state interactions. For the time independent case, which we treat here, the Lax-Phillips $S$-matrix, according to (4.1), can be written as

$$
S(\sigma)=\lim _{t \rightarrow \infty} e^{i \mathrm{H}_{0}(\sigma) t} e^{-2 i \mathrm{H}(\sigma) t} e^{i \mathrm{H}_{0}(\sigma) t},
$$

where $\mathrm{H}(\sigma)=U^{\dagger}(\sigma) \mathrm{H} U(\sigma)$ and $\mathrm{H}_{0}(\sigma)=U^{\dagger}(\sigma) \mathrm{H}_{0} U(\sigma)$. We then construct $U(\sigma)$ so that it induces a diffeomorphism* on the spectrum of the unperturbed Hamiltonian in such a

* We thank G. Goldin for a discussion of this point. 
way that, for $\mathrm{H}_{0}|\lambda\rangle=\lambda|\lambda\rangle$,

$$
U(\sigma)|\lambda\rangle=|\lambda\rangle_{\sigma}=|\lambda(\sigma)\rangle \sqrt{\Lambda(\sigma, \lambda)},
$$

where

$$
\Lambda(\sigma, \lambda)=\frac{d \lambda(\sigma)}{d \sigma} .
$$

The factor of the square root of the Jacobian is necessary to assure that $\left\langle\lambda \mid \lambda^{\prime}\right\rangle=\delta\left(\lambda-\lambda^{\prime}\right) \equiv$ $\left\langle\lambda\left|U^{\dagger}(\sigma) U(\sigma)\right| \lambda^{\prime}\right\rangle$. It follows that $\mathrm{H}_{0}(\sigma)|\lambda\rangle=\lambda(\sigma)|\lambda\rangle$, where $\lambda \rightarrow \lambda(\sigma)$ is a smooth map which can be analytically continued. The procedure outlined above in (4.2)-(4.10), applied to the auxiliary space problem, then results in

$$
\begin{aligned}
& \left\langle\lambda|S(\sigma)| \lambda^{\prime}\right\rangle=\delta\left(\lambda-\lambda^{\prime}\right) \\
& \quad-2 \pi i \delta\left(\lambda(\sigma)-\lambda^{\prime}(\sigma)\right)\left\langle\lambda\left|T_{\sigma}^{a u x}(\lambda(\sigma)+i \epsilon)\right| \lambda^{\prime}\right\rangle,
\end{aligned}
$$

where

$$
\begin{aligned}
T_{\sigma}^{a u x}(z) & =V_{\sigma}+V_{\sigma} G_{\sigma}^{a u x}(z) V_{\sigma} \\
V_{\sigma} & =U^{\dagger}(\sigma) V U(\sigma)
\end{aligned}
$$

and

$$
G_{\sigma}^{a u x}(z)=U^{\dagger}(\sigma) G^{a u x}(z) U(\sigma) .
$$

Here, $G^{\text {aux }}(z)=(z-H)^{-1}$ is a resolvent kernel in the auxiliary space, and we have written (5.4) in terms of operator-valued matrix elements, suppressing the degeneracy indices (such as angular variables).

Since $\lambda \rightarrow \lambda(\sigma)$ is $1: 1$ for each $\sigma$, we may write (5.4) as

$$
\left\langle\lambda|S(\sigma)| \lambda^{\prime}\right\rangle=\delta\left(\lambda-\lambda^{\prime}\right)\left\{1-\frac{2 \pi i}{\Lambda(\sigma, \lambda)}\left\langle\lambda\left|T_{\sigma}(\lambda(\sigma)+i \epsilon)\right| \lambda\right\rangle\right\},
$$

The relations (5.1)-(5.7) are valid for any $t$-independent non-relativistic scattering problem (for which $U(\sigma)$ induces a diffeomorphism). For the Lee-Friedrichs ${ }^{15}$ model, in particular, since the set $\{|\lambda(\sigma)\rangle\}$ is complete on the continuum, if we assume that the bound state embedded in the continuum is non-degenerate, the action of $U(\sigma)$ in the discrete eigenstate is just a phase, i.e.,

$$
U(\sigma) \phi=u_{0}(\sigma) \phi,
$$

where $u_{0}(\sigma)$ is a smooth complex valued function of $\sigma$ with unity modulus. Then, since in this model, $\left\langle\lambda\left|V_{\sigma}\right| \lambda^{\prime}\right\rangle=0$,

$$
\left\langle\lambda\left|V_{\sigma} G_{\sigma}(\lambda(\sigma)+i \epsilon) V_{\sigma}\right| \lambda\right\rangle=\Lambda(\sigma, \lambda) W(\lambda(\sigma)) R_{\sigma}(\lambda(\sigma)+i \epsilon),
$$

where (the modulus squared implies a sum over degeneracy parameters as well)§

$$
W(\lambda(\sigma))=(\Lambda(\sigma, \lambda))^{-1}\left|\left\langle\lambda\left|V_{\sigma}\right| \phi\right)\right|^{2}=|\langle\lambda(\sigma)|V| \phi)|^{2}
$$

$\S$ We use round brackets for scalar products with proper (auxiliary) Hilbert space vectors, and angular brackets for generalized states on the continuum. 
and

$$
R_{\sigma}(z)=\left(\phi\left|\frac{1}{\lambda(\sigma)-H(\sigma)+i \epsilon}\right| \phi\right) \equiv\left(\phi\left|G_{\sigma}(\lambda(\sigma)+i \epsilon)\right| \phi\right)
$$

Using the second resolvent equation in the form

$$
G_{\sigma}(z)=G_{\sigma}^{0}(z)+G_{\sigma}^{0}(z) V_{\sigma} G_{\sigma}(z)
$$

where

$$
G_{\sigma}^{0}(z)=\frac{1}{\lambda(\sigma)-H_{0}(\sigma)+i \epsilon}
$$

one obtains

$$
\begin{aligned}
& \left(\phi\left|G_{\sigma}(\lambda(\sigma)+i \epsilon)\right| \phi\right)= \\
& \quad=\frac{1}{\lambda(\sigma)-E_{0}+i \epsilon}+\frac{1}{\lambda(\sigma)-E_{0}+i \epsilon} \int d \lambda^{\prime}\left(\phi\left|V_{\sigma}\right| \lambda^{\prime}\right\rangle\left\langle\lambda^{\prime}\left|G_{\sigma}(\lambda(\sigma)+i \epsilon)\right| \phi\right)
\end{aligned}
$$

and

$$
\left\langle\lambda^{\prime}\left|G_{\sigma}(\lambda(\sigma)+i \epsilon)\right| \phi\right)+\frac{1}{\lambda(\sigma)-\lambda^{\prime}(\sigma)+i \epsilon}\left\langle\lambda^{\prime}\left|V_{\sigma}\right| \phi\right)\left(\phi\left|G_{\sigma}(\lambda(\sigma)+i \epsilon)\right| \phi\right) .
$$

Substituting (5.15) into (5.14), we obtain a formula for the reduced resolvent

$$
\begin{aligned}
R_{\sigma}(\lambda(\sigma)+i \epsilon) & =\left(\lambda(\sigma)+i \epsilon-E_{0}-\int d \lambda^{\prime} \Lambda(\sigma, \lambda) \frac{W\left(\lambda^{\prime}(\sigma)\right)}{\lambda(\sigma)-\lambda^{\prime}(\sigma)+i \epsilon}\right)^{-1} \\
& =\left(\lambda(\sigma)+i \epsilon-E_{0}-\int d \lambda^{\prime}(\sigma) \frac{W\left(\lambda^{\prime}(\sigma)\right)}{\lambda(\sigma)-\lambda^{\prime}(\sigma)+i \epsilon}\right)^{-1}
\end{aligned}
$$

since

$$
d \lambda^{\prime} \Lambda\left(\sigma, \lambda^{\prime}\right)=d \lambda^{\prime}(\sigma)
$$

The condition $S(\sigma) n(\lambda)$ have a co-dimension in the auxiliary space is then that there exist some (measurable) $m(\lambda)$, such that (the factor $\Lambda^{-1}$ in (5.7) cancels the factor $\Lambda$ of $(5.9)$ )

$$
\int d \lambda d \lambda^{\prime} m(\lambda)^{*}\left\langle\lambda|S(\sigma)| \lambda^{\prime}\right\rangle n\left(\lambda^{\prime}\right)=0
$$

for all $n(\lambda)$. Hence,

$$
\left.\left\{1-2 \pi i W(\lambda(\sigma)) R_{\sigma}(\lambda(\sigma)+i \epsilon)\right)\right\}^{*} m(\lambda)=0
$$

For the case of a non-degenerate spectral model, for which $m(\lambda)$ is a one-dimensional function of $\lambda$, for every $\lambda$ in the support of $m(\lambda)$, the expression in brackets must vanish, i.e., we must have

$$
\lambda(\sigma)+i \epsilon-E_{0}-\int_{0}^{\infty} d \lambda^{\prime}(\sigma) \frac{W\left(\lambda^{\prime}(\sigma)\right)}{\lambda(\sigma)-\lambda^{\prime}(\sigma)+i \epsilon}=2 \pi i W(\lambda(\sigma)) .
$$


Changing variables, $\lambda^{\prime}(\sigma) \rightarrow \lambda^{\prime}$, we can write the condition (5.19) as

$$
\lambda(\sigma)+i \epsilon-E_{0}-\int_{0}^{\infty} d \lambda^{\prime} \frac{W\left(\lambda^{\prime}\right)}{\lambda(\sigma)-\lambda^{\prime}+i \epsilon}=2 \pi i W(\lambda(\sigma)) .
$$

Note that the right hand side would precisely cancel with the jump contribution if we crossed the cut in the integral from above, interpreting $\lambda(\sigma) \rightarrow \zeta$ as a complex variable. Taking the imaginary part of (5.20) in the form

$$
\zeta+i \epsilon-E_{0}-\int_{0}^{\infty} d \lambda^{\prime} \frac{W\left(\lambda^{\prime}\right)}{\left(\zeta-\lambda^{\prime}\right)}=2 \pi i W(\zeta),
$$

one finds, as the the usual treatment of the Lee-Friedrichs model ${ }^{21}$ (numerical studies were carried out in ref. 22),

$$
\operatorname{Im} \zeta\left(1+\int d \lambda^{\prime} \frac{W\left(\lambda^{\prime}\right)}{\left|\zeta-\lambda^{\prime}\right|^{2}}\right) \cong W(\zeta)
$$

where we have assumed $\operatorname{Im} \zeta$ small, and $W(\zeta)$ analytic in some neighborhood of the real axis, and primarily real for $\operatorname{Im} \zeta$ small. The Eq. (5.22) therefore may have a solution in the first sheet of the upper half plane. As in dilation analytic theories ${ }^{18,19}$, the Lax-Phillips method has effectively moved the cut below the root. For the value of $\sigma$ admitting a null space, and for a value of $\lambda=\lambda_{0}$ that satisfies (5.20), there must be a finite interval in the neighborhood of $\lambda_{0}$ that also satisfies this condition so that $m(\lambda)$ can have measurable support. Hence, the first derivative of the condition (5.20) must be valid.

Taking the derivative of (5.20), one obtains

$$
\Lambda(\sigma, \lambda)\left\{1+\int d \lambda^{\prime} \frac{W\left(\lambda^{\prime}\right)}{\left(\lambda(\sigma)-\lambda^{\prime}\right)^{2}}-2 \pi i W^{\prime}(\lambda(\sigma))\right\}=0 .
$$

For sufficiently small coupling (in the generic case, for which the expression in brackets does not, by a special analytic structure, vanish), reflected by the size of $W(\lambda)$, the condition (5.23) cannot be satisfied unless the map induced by $U(\sigma)$ has a critical point, i.e.,

$$
\Lambda(\sigma, \lambda)=\frac{d \lambda(\sigma)}{d \lambda}=0
$$

at the point $\zeta$ satisfying the condition (5.21).

The function $\lambda(\sigma)$ is a monotonic and smooth function on the real line, but its analytic continuation in $\sigma$ can have such properties. For $\lambda(\sigma)$ in the neighborhood of the solution $\zeta_{0}$ of $(5.21)$, the form

$$
\lambda(\sigma) \cong \frac{\lambda_{0}^{2}}{\sigma}+\mathrm{O}\left(\left(\lambda-\lambda_{0}\right)^{2}\right)
$$

satisfies the conditions (5.21) and (5.24) for $\sigma=\zeta_{0}^{*}, \lambda=\lambda_{0}=\left|\zeta_{0}\right|$. In this simple one-dimensional illustration, the function $m(\lambda)$ is characterized only by its local support property on the spectrum of $\mathrm{H}_{0}$. It would then follow from (5.20) and (5.22) that the Lax-Phillips exponential law goes precisely with the pole of the Lee-Friedrichs model. 


\section{Conclusions and Discussion}

An exact semigroup evolution law (exponential decay), corresponding to an irreversible process, can be achieved within the framework of a microscopic quantum theory if the Hilbert space carries a natural foliation along an axis in its measure space on which the wave function moves by translation, under the full unitary evolution, in a special class of (translation) representations. The foliation of such a space is assured by a theorem of Sinai ${ }^{14}$ when there are distinguished incoming and outgoing subspaces $\mathcal{D}_{ \pm}$which are stable under forward (backward) unitary evolution. Lax and Phillips developed a complete theory of such systems for the case of classical hyperbolic (wave) equations for scattering on a bounded target ${ }^{1}$. Flesia and Piron ${ }^{2}$ showed that the quantum mechanical Hilbert space can be extended, by a direct integral construction over the time variable, to form a structure in which the Lax-Phillips theory can be applied. In a succeeding study ${ }^{4}$, it was shown that a necessary condition for a non-trivial Lax-Phillips theory, for which the singularities of the $S$-matrix in the spectral variable constitute the spectrum of the generator of the semigroup, is that the evolution operator act as a smooth (operator-valued) integral kernel on the time axis in the free translation representation. We have shown in this paper that a pointwise (in $t$ ) dynamical evolution operator in what we have called the model representation, in which the Hamiltonian of a system and the time variable appear with their usual laboratory interpretation, maps into a smooth, non-trivial kernel in the free translation representation, and therefore satisfies this necessary condition.

We have shown that the subspaces $\mathcal{D}_{ \pm}$may be constructed from the wave operators, intertwining the full and unperturbed Lax-Phillips evolution operators, applied to functions with definite half-line support properties on the $t$-axis. The orthogonality of these subspaces follows from the analytic properties of the $S$-matrix.

We have furthermore shown that the Lax-Phillips $S$-matrix is equivalent to the $S$ matrix of the standard scattering theory (for the time dependent case as well) by a unitary transformation which is parametrized by the Lax-Phillips spectral variable. This unitary transformation arises from the transformation from the model representation to the free spectral representation (the Fourier transform of the free translation representation). There is considerable freedom in choosing such a function, which has the property, upon analytic continuation to the upper half-plane, of bringing the $S$-matrix to a form in which there is a non-trivial null co-space, corresponding to the eigenvectors of the resonant state (these points are conjugate to the resonant poles in the lower half plane). Since these vectors lie in the (auxiliary) Hilbert space, they may be used to compute expectation values of the usual dynamical variables, such as position, momentum, or angular momentum. Such properties are not available for the generalized functions obtained in the method of constructing Gel'fand triples ${ }^{13}$ or the dilation analytic methods ${ }^{18,19}$.

The work of Lee, Oehme and Yang ${ }^{9}$ and Wu and Yang ${ }^{9}$, assuming an effective Hamiltonian analogous to the Wigner-Weisskopf pole approximation in the form of a two-by-two non-Hermitian matrix, results in an exact semigroup structure. As has been pointed out ${ }^{8}$, deviations due to a more accurate treatment of the Wigner-Weisskopf method, reflecting its non-semigroup structure, could be important in regeneration processes; if, however, as the experimental results on $K$-meson decay $^{11}$ seem to imply, the phenomenological parameterization of refs. 9 are indeed consistent to a high level of accuracy, the Lax-Phillips 
theory could provide a microscopic framework for this exact semigroup behavior.

We gave here an illustration of the method for a one channel non-degenerate LeeFriedrichs model ${ }^{15,21}$ for the underlying dynamics. The illustration was worked out by assuming, for simplicity, that the unitary transformation relating the Lax-Phillips $S$-matrix and that of the usual quantum scattering induces a diffeomorphism on the continuous spectrum without altering the spectral family; upon analytic continuation, we showed that the resulting Lax-Phillips semigroup can be generated by the complex pole of the usual Lee-Friedrichs model, and a measurable eigenfunction in the Hilbert space can be found for the null co- space of the $S$-matrix at this point. A more detailed analysis of this model, as well as other applications, for example, to the two channel problem (e.g., $K$ meson decay), atomic and molecular and condensed matter physics, will be discussed elsewhere.

We finally remark that the $\tau$-dependent decay law of the unstable system can be calculated, as we have pointed out in Eq.(1.9), in terms of the full evolution acting on a state in $\mathcal{K}$. The Laplace transform of this amplitude then exhibits the full Green's function $(z-K)^{-1}$, which can be expanded in an infinite series, with the help of (4.7), as was done for the $S$-matrix in Section 4 . The Laplace transform of the amplitude can then be expressed in terms of the Lax-Phillips $S$-matrix, realizing the theorem of Lax and Phillips ${ }^{1}$ cited in Section 1. The details of the demonstration of this result and its applications will also be given elsewhere.

\section{Acknowledgements}

One of us (L.H.) wishes to thank S.L. Adler, C. Newton, C. Piron and T.T. Wu for the opportunity to discuss the structure of the Lax-Phillips theory (and to express his appreciation to Professor Adler for his hospitality at the Institute for Advanced Study where the work reported here was started), B. Altshuler and O. Agam for their encouragement and discussions of possible applications to condensed matter physics, and I. Dunietz, Y.B. Hsiung, and B. Winstein and other colleagues at the Fermilab for many discussions of the implications of the current experiments on $K$ meson decay as well as potential applications in $B$ physics for the theoretical structure that we have developed here.

\section{References}

1. P.D. Lax and R.S. Phillips, Scattering Theory, Academic Press, N.Y. (1967).

2. C. Flesia and C. Piron, Helv. Phys. Acta 57, 697 (1984).

3. L.P. Horwitz and C. Piron, Helv. Phys. Acta 66, 694 (1993).

4. E. Eisenberg and L.P. Horwitz, in Advances in Chemical Physics, XCIX, p. 245, ed. I. Prigogine and S. Rice, John Wiley and Sons, N.Y. (1997).

5. C. Piron, Foundations of Quantum Physics, Benjamin/Cummings, Reading, Mass. (1976).

6. V.F. Weisskopf and E.P. Wigner, Zeits. f. Phys. 63, 54 (1930); 65, 18 (1930).

7. L.P. Horwitz, J.P. Marchand and J. LaVita, J. Math. Phys. 12, 2537 (1971); D. Williams, Comm. Math. Phys. 21, 314 (1971).

8. L.P. Horwitz and L. Mizrachi, Nuovo Cimento 21A, 625 (1974).

9. T.D. Lee, R. Oehme and C.N. Yang, Phys. Rev. 106, 340 (1957); T.T. Wu and C.N. Yang, Phys. Rev. Lett. 13, 380 (1964). 
10. G. Gamow, Z. Phys. 51, 204(1928).

11. B. Winstein, et al, Results from the Neutral Kaon Program at Fermilab's Meson Center Beamline, 1985-1997, FERMILAB-Pub-97/087-E, published on behalf of the E731, E773 and E799 Collaborations, Fermi National Accelerator Laboratory, P.O. Box 500, Batavia, Illinois 60510.

12. S.R Wilkinson, C.F. Bharucha, M.C. Fischer, K.W. Madison, P.R. Morrow, Q. Niu, B. Sundaram, and M. Raizen, Nature 387, 575 (1997)

13. W. Baumgartel, Math. Nachr. 69, 107 (1975); L.P. Horwitz and I.M. Sigal, Helv. Phys. Acta 51, 685 (1978); G. Parravicini, V. Gorini and E.C.G. Sudarshan, J. Math. Phys. 21, 2208 (1980); A. Bohm, Quantum Mechanics: Foundations and Applications, Springer, Berlin (1986); A. Bohm, M. Gadella and G.B. Mainland, Am. J. Phys. 57, 1105 (1989); T. Bailey and W.C. Schieve, Nuovo Cimento 47A, 231 (1978).

14. I.P. Cornfield, S.V. Formin and Ya. G. Sinai, Ergodic Theory, Springer, Berlin (1982).

15. K.O. Friedrichs, Comm. Pure Appl. Math. 1, 361 (1948); T.D. Lee, Phys. Rev. 95, 1329 (1956).

16. J.S. Howland, Springer Lecture Notes in Physics,, vol. 130, p. 163, Springer, Berlin (1979).

17. T. Kato, Perturbation Theory for Linear Operators, Springer, N.Y. (1966).

18. J. Aguilar and J.M. Combes, Comm. Math. Phys. 22, 280 (1971); J.M. Combes, Proc. Int. Cong. of Math., Vancouver (1974).

19. B. Simon, Ann. Math. 97, 247 (1973).

20. For example, J.R. Taylor, Scattering Theory, John Wiley and Sons, N.Y. (1972); R.J. Newton, Scattering Theory of Particles and Waves, McGraw Hill, N.Y. (1976).

21. L.P. Horwitz and J.P. Marchand, Rocky Mountain J. of Math. 1, 225 (1971).

22. N. Bleistein, H. Neumann, R. Handelsman and L.P. Horwitz, Nuovo Cimento 41A, 389 (1977). 